\title{
Les multiples visages de l'urgence : point de vue sur les travaux
}

Yves Jeanneret

\section{OpenEdition}

1 Journals

Édition électronique

URL : http://journals.openedition.org/communicationorganisation/3419

DOI : 10.4000/communicationorganisation.3419

ISSN : 1775-3546

Éditeur

Presses universitaires de Bordeaux

\section{Édition imprimée}

Date de publication : 1 juin 2006

Pagination : 235-241

ISSN : $1168-5549$

\section{Référence électronique}

Yves Jeanneret, "Les multiples visages de l'urgence : point de vue sur les travaux », Communication et organisation [En ligne], 29 | 2006, mis en ligne le 21 juin 2012, consulté le 22 décembre 2020. URL: http://journals.openedition.org/communicationorganisation/3419; DOI : https://doi.org/10.4000/ communicationorganisation.3419

Ce document a été généré automatiquement le 22 décembre 2020.

(c) Presses universitaires de Bordeaux 


\title{
Les multiples visages de l'urgence : point de vue sur les travaux
}

\author{
Yves Jeanneret
}

1 Pour qui revient sur la diversité et l'ampleur spectaculaires des questions débattues pendant ces deux journées, un premier constat s'impose. Alors que le terme d'urgence propose spontanément à l'esprit, dans sa petite mythologie ordinaire, l'idée d'une concentration, d'une fulgurance, la réflexion sur les rapports entre urgence et organisation, dès lors qu'elle s'appuie sur une approche attentive des pratiques, manifeste une épaisseur considérable des médiations. Le colloque a peuplé et ainsi, inévitablement, élargi, la ponctualité apparente de la situation d'urgence. Il l'a fait en manifestant la pluralité des figures possibles de l'urgence.

2 Ceci me suggère une comparaison entre ce colloque et un autre, organisé il y a quelques années à l'initiative de Frédéric Lambert sur Figures de l'anonymat, médias et société1. Dans les deux cas, l'analyse des figures produit son effet, révélant ce que l'attention ordinaire ne discerne pas. L'anonymat, qui paraît consister en une simple absence, émerge de toute une construction positive; l'urgence, qui paraît relever d'une immédiateté pure, est affaire de médiations.

D'abord, on ne soumet pas impunément à une communauté interdisciplinaire de chercheurs le terme «figures». Figure est entendu, dans les communications du colloque, d'abord comme figuration. C'est l'émergence d'une forme, entre l'événement qui surgit, inattendu, indécis, incertain et les constructions sociales qui marquent les cadres dans lesquels il va être perçu, rationalisé, maîtrisé, instrumentalisé : temps, espace, action, valeur. J'y reviens. Mais figure s'entend aussi au bon sens classique de la figure rhétorique, car l'urgence apparaît au fil des travaux ici présentés comme un incontrôlable élaboré, domestiqué, nommé, normé. Les hommes formulent médiatisent, désignent, formatent, procédurent si l'on peut dire les situations d'urgence. On pense à ce dessin de Jacques Faizant publié dans le Figaro aux riches heures du gaullisme, où on voit le Général, orfèvre comme on le sait en conférences de presse, trôner devant un bureau couvert de fiches, qui déclare fièrement à son Premier Ministre : «Vous voyez, 
Pompidou, ce qu'il y a de terrible avec l'improvisation, c'est tout le travail préparatoire $»$.

4 L'une des catégories de figures qui ressortent avec un particulier relief du vocabulaire employé pendant ces journées et des situations qui y ont été évoquées, c'est ce qui ressortit à la théâtralité. L'urgence, c'est le nœud, la catastrophe, la péripétie. C'est la crise (non nommée comme telle, j'y reviens). Mais c'est surtout la dimension héroïque de l'action.

5 C'est pourquoi une autre surprise de ces journées - qui a beaucoup agité les échanges informels dans les pauses - c'est la force et en même temps la multiplicité des références normatives que semble inévitablement convoquer l'idée d'urgence. Polarités très fortes, mais polarités fortement hérérogènes. Urgere ou emergere, selon qu'on choisit la référence française ou anglaise, ne définissent pas d'abord l'urgence par une coordonnée temporelle, mais par une situation de confrontation, à la pression qu'exerce sur nous le réel ou à l'incursion qu'il fait dans notre vie. Etre dans l'urgence, c'est, comme nous l'avons indiqué avec Cécile Tardy, être sujet de passion devant l'action, être en situation de subir une situation où il faut agir. L'imaginaire qui entoure l'idée d'urgence, c'est celui d'une axiologie pressante dotée d'un relief extrême. L'urgence, c'est ce qui nous réclame instamment.

6 L'action, et avec elle ses normes et ses finalités, est donc omniprésente dans tout travail de recherche sur l'urgence, prise en compte, commentée, subie, accompagnée, devancée selon les cas. L'idée que l'urgence convoque le rapport à l'action et les normes de l'action dans la recherche aura sans doute été omniprésente dans le colloque. Toutefois, cette convocation des normes s'est faite selon trois grandes figures, sans doute en grande part incompatibles entre elles. Dans une première interprétation, l'urgence est un fait de contemporanéité, contre lequel il faut avant tout se prémunir : le moment actuel participe à une idéologie du temps efficace, contracté, réactif, où on peut voir la marque de l'aliénation contemporaine. Dans une seconde perspective, l'urgence est une donnée anthropologique fondamentale et à quelque titre permanente. L'urgence tient à la présence du drame dans l'existence humaine, à la pression de l'histoire sur les désarrois et les abandons, et elle appelle une mobilisation qui, même lorsqu'elle est professionnellement assumée, relève de l'émotion et de l'engagement. Enfin, une troisième figure parcourt, plus secrètement, beaucoup d'analyses ; elle fait de l'urgence essentiellement une trace de l'ingérable et du désordre. Je rappelle en effet que c'est par la notion d'urgence que Foucault définissait la catégorie, si importante à ses yeux, de dispositif : "par dispositif, déclarait-il dans un échange avec des amis psychanalystes, j'entends une sorte - disons - de formation, qui, à un moment historique donné, a eu pour fonction majeure de répondre à une urgence. Le dispositif a donc une fonction stratégique dominante $»^{2}$. Urgence, c'est-à-dire ici, dans le cadre de la pensée du pouvoir disséminé et sans maître, situation chargée d'enjeux et impossible à maîtriser. Par exemple, le spectacle des sacrifices sur lequel la justice royale pouvait trôner porte la menace d'une enflammation du peuple, à laquelle les disciplines de la douceur pénale vont remédier, sans vraiment le contrôler. Donc, si on mène le raisonnement à son terme, ces communications où l'urgence relève d'une stratégie de l'incontrôlé sont porteuses d'une approche des relations de communication dans le travail ou la politique où, finalement, c'est la notion même d' "organisation » qui se trouve contestée. Urgence et communication organisationnelle seraient donc unies, non par une complémentarité, mais par une exclusion mutuelle? 
7 Trois figures de la culture de masse pourraient incarner ces axiologies de l'urgence : Charlot vissant compulsivement ses boulons dans Les temps modernes, L'abbé Pierre interpellant les auditeurs de radio-Luxembourg, René Victor Pilhes débusquant dans son roman l'imprécateur les fissures de l'édifice managérial.

8 Au fil de ces trois interprétations de la valeur de l'urgence, ce sont des axiologies très vives, mais très contrastées, qui sont mobilisées par les chercheurs qui se sont succédé pendant ces deux jours, la véhémence étant égale à stigmatiser l'urgence, à la requérir, à la revendiquer. Univers racinien en somme, où l'on peut passer de l'amour à la haine, mais qui ne connaît guère d'indifférence. L'urgence mérite-t-elle cet excès d'honneur et cette indignité?

9 Voici qui nous mène, logiquement, à la complexité des définitions possibles de l'urgence elle-même, complexité qui révèle beaucoup de jalons pour des recherches futures. Elle se marque d'abord par la richesse et l'ambiguïté de la constellation imaginaire qu'appelle avec elle l'idée d'urgence. L'urgence a été très souvent associée à l'idée d'une connexion et d'une disponibilité, d'une présence à l'autre, qui est très ambivalente, parce qu'elle est un élément de pouvoir et une figure de la dépendance. Mais il y a surtout le cortège des notions si proches et pourtant - plus d'un l'a souligné nettement - distinctes de l'urgence: disponibilité, immédiateté, instantanéité, événementialité.

10 La difficulté que nous éprouvons à saisir l'urgence tient aussi à la diversité considérable des perspectives temporelles à partir desquelles l'urgence peut être observée, puisqu'elle manifeste, selon une formule proposée, un «temps complexe». Il y a, par exemple, dans l'idée de développement durable, une conception de l'urgence du temps long, qui nous arrache tout à fait à l'évidence du chronomètre. Mais beaucoup de situations décrites, où les moments critiques se multiplient, relèvent de ce que Bernadette Dufrêne appelle "les répliques de l'événement», empruntant cette métaphore à la sismologie ${ }^{3}$.

11 Cette complexité diachronique de l'urgence se double d'une complexité diachronique, et qu'on pourrait résumer par le fait que l'urgence, dans le donné des situations qu'elle nous impose, radicalise les points de vue sur le processus de communication. Il est aisé de montrer que dans les diverses analyses proposées pendant ces deux jours, le point de vue du chercheur et la nature du sujet considéré placent souvent un niveau de la communication en situation hégémonique, jusqu'à estomper, miner ou absorber tous les autres. Dans le monstre communicationnel de l'urgence (monstrum; l'objet qui exhibe tout à coup un prodige), tout semble relationnel pour certains, tout technique pour d'autres, tout tactique pour d'autres encore.

12 Ce phénomène d'implosion de l'idée d'urgence en une pluralité de situations d'urgence - comme si l'urgence changeait de visage et pas seulement de figure - n'est pas sans rapport avec le fait que, dans le vaste espace de la communication organisationnelle, toutes les activités ne rencontrent pas l'urgence de la même manière. Certaines activités, certains métiers, certains engagement, se définissent centralement par leur relation à l'urgence : celle-ci apparaît dans ce cas comme un champ spécifique de la communication organisationnelle. Mais, parallèlement, on observe une sorte de dissémination de la question de l'urgence, sous des modalités diffuses, dans toutes les activités sociales. L'urgence serait donc quelque chose comme une épistémè de la gestion contemporaine. Enfin, il y a des scénographies de l'urgence, un spectacle médiatique de l'urgence, une mise en visibilité gestionnaire de l'urgence, une 
sensibilisation sociale à l'urgence, qui se rattachent particulièrement à une rhétorique du temps vécu. Les anciens avaient leur laudatio temporis acti (éloge des temps passés) qui colorait de nostalgie - qui, comme on le sait, n'est plus ce qu'elle était - la perception de l'histoire. Nous avons un sentiment de l'urgence, que par exemple l'antienne du «retard français» nous rappelle périodiquement - car la France est toujours en retard d'un retard, chacun le sait.

Evidemment tout cela nous suggère une question que nous ne pouvons pas éluder, et que je me garderai bien de trancher : est-ce bien de la même chose que nous parlons dans tous ces cas lorsque nous évoquons l'urgence? En d'autres termes, où se trouve l'articulation entre l'idée d'urgence, l'idée de communication et l'idée d'organisation?

Ces lignes de force trop rapidement dessinées - il s'agit bien de force, on sent que la question mobilise de l'énergie - ne vont pas sans faire évidemment retour sur la recherche en communication elle-même. Les SIC sont peut-être aujourd'hui placées devant une sorte d'instance de l'urgence. La question de l'urgence ne constitue pas seulement pour la recherche un objet, mais un enjeu. Avant tout, l'urgence fournit aux analyses un sol techno-sémiotique très fertile, de nombreuses interventions le montrent amplement : la complexité des rapports que la situation d'urgence développe entre le logistique et le symbolique est une occasion d'exercer l'analyse communicationnelle avec finesse. Mais l'urgence est aussi pour les SIC une occasion de justification. Celles-ci y trouvent matière à montrer leur disponibilité, à répondre par des dispositifs - puisque les dispositifs sont, on l'a vu, une réponse à des urgences - à certaines difficultés de la société, et plusieurs courants de la discipline se sont engagés dans ce sens.

Un pas de plus, et les SIC pourraient être sommées, dans le contexte de la valeururgence, à rendre des comptes sur ce que la recherche peut apporter, tout de suite, sans délai, aux urgences du temps. J'en ai fait souvent l'expérience, dans le domaine de l'édition et de l'écriture, où nombreux sont les acteurs qui considèrent toute prise de recul, toute prudence, tout jugement nuancé sur l'investissement des réseaux comme une forme de trahison d'un idéal qui ne saurait attendre : la présence du français sur internet, la valorisation de la discipline, la défense de certaines idées, l'établissement d'une démocratie participative. Ce que Nicolas Auray nomme l' "ébranlement critique ${ }^{4}$, cette nécessité absolue de demédier à une situation intolérable, est omniprésente dans ce secteur. La question est évidemment: dans quelle mesure la recherche peut-elle se penser elle-même comme une activité urgente? Nos prédécesseurs auraient répondu, il y a quelques décennies, négativement à cette question sans hésitation. Saurions-nous y répondre aujourd'hui?

Bref. La série des colloques sur l'urgence mérite de continuer. Beaucoup de questions restent pleinement ouvertes. Il me semble d'abord que, compte tenu de l'importance donnée dans nos échanges à l'histoire des cultures du temps, il serait important d'engager un dialogue interdisciplinaires avec les historiens de l'antiquité, des cultures non européennes, du Moyen-Age, parce que la complexité des cultures du temps de ces civilisations est bien autre que ce qu'a pu faire apparaître, rétrospectivement, la conception que les " postmodernes » ont pu élaborer d'un passé à leurs yeux linéaire. Mais j'aimerais évoquer, pour finir, quelques perspectives de recherche en SIC qui ne se trouvent pas avoir été mentionnées ici, mais qui pourraient prolonger les questions qui ont été posées. 
17 J'observe d'abord qu'il n'a pas été question dans ces journées de l'idée de « communication de crise ». Pourtant, le sujet qu'abordait par exemple Marie-Noelle Sicard dans son livre sur les crises technologiques ${ }^{5}$ était omniprésent et la notion de communication de crise a beaucoup été travaillée à Bordeaux. Peut-être le fait de penser ce qui est en jeu en termes d'urgence est-il incompatible avec le fait de le définir comme une crise? Je laisse la question ouverte. Il me semble qu'une réflexion sur cette redéfinition forte des objets et ce qu'elle signifie serait très intéressante.

Je pensais aussi aux réflexions conduites par Bernard Lamizet ${ }^{6}$ sur ce qui relève, dans la communication, du réel, de l'imaginaire et du symbolique : elles me semblent pouvoir sérieusement nourrir ce qui a été discuté ici, car l'urgence est précisément une façon de définir la situation de communication dans une perspective qui associe nécessairement ces trois instances (en d'autres termes de ne pas se situer dans une perspective exclusivement constructiviste, l'urgence c'est un peu le réel qui frappe à la porte, ce dont on ne sait pas bien ce qu'on peut dire). Pour avancer dans une sémiotique de l'urgence, il faut reconsidérer le poids du réel dans l'ordre des signes.

Enfin, par-delà l'hypothèse d'une soumission générale des organisations à un impératif managérial de l'urgence, il y a encore beaucoup à faire pour mieux comprendre comment se traduit concrètement cette idée d'urgence en termes de dispositifs de communication et quelle est la définition du pouvoir qui y est en jeu. Je signale à cet égard la thèse défendue par Sophie Pène que nous entrons, après la société de surveillance, dans une "société de disponibilité " ${ }^{7}$, associant de façon nouvelle une certaine omniprésence de l'écriture dans les organisations, une mise en visibilité de toutes les actions et un régime des savoirs où l'interaction constitue en permanence la ressource possible d'un avantage concurrentiel.

\section{NOTES}

1. Lambert, F., dir., Figures de l'anonymat, médias et société, actes du colloque tenu à l'ENS Saint-Cloud en juin 1999, Paris, L'Harmattan, 2001.

2. Foucault, "Le jeu de Michel Foucault", Ornicar, n 10, juillet 1977, repris dans Dits et écrits, 1976-1988, Gallimard, 1994, p. 302.

3. Dufrêne, Bernadette, Evénement et processus de communicaiton : qu'est-ce qu'un événement culturel international ? Mémoire d'HDR, Paris 4, 2003.

4. Auray, Nicolas, Politiques de l'informatique et de l'information: les pionniers de la nouvelle frontière électronique, thèse, EHESS, 2000.

5. Sicard, Marie-Noelle, Entre médias et crises technologiques : les enjeux communicationnels, Villeneuve d'Ascq, Presses du Septentrion, 1998.

6. Lamizet, Bernard, Les poussières de l'histoire: une sémiotique de l'événement, ouvrage à paraître prochainement.

7. Pène, Sophie, Société de disponibilité : la vie quotidienne des communautés artificielles. HDR, Paris 4, 2005. 


\section{AUTEUR}

\section{YVES JEANNERET}

Mail : yves.jeanneret-celsa@paris4.sorbonne.fr 